\section{REFERENCES}

[1] KAMMASH, T., GalbRaITH, D.L., Nucl. Fusion 29 (1989) 1079.

[2] KAMMASH, T., GALBRAITH, D.L., J. Br. Interplanet. Soc. 41 (1988) 527.

[3] HASEGAWA, A., NISHIHARA, K., DAIDO, H., et al., Nucl. Fusion 28 (1988) 369.

[4] MEYER-TER-VEHN, J., Nucl. Fusion 22 (1982) 561.
[5] LINDL, J.D., MARK, J.W.-K., Laser Part. Beams 3 (1985) 37.

[6] STORM, E., BATHA, S.H., BERNAT, T.P., et al., in Plasma Physics and Controlled Nuclear Fusion Research 1990 (Proc. 13th Int. Conf. Washington, DC, 1990), Vol. 3, IAEA, Vienna (1991) 99

[7] TAKABE, H., MIMA, K., Numerical Study of Ignition by Stagnation-Free Implosion, Res. Rep. ILE 8713P, Institute of Laser Engineering, Osaka University (1987).

\title{
REPLY TO \\ COMMENTS BY H. NAKASHIMA, H. TAKABE \\ ON THE PAPER BY T. KAMMASH, D.L. GALBRAITH \\ 'A HIGH GAIN FUSION REACTOR BASED ON THE MAGNETICALLY INSULATED INERTIAL CONFINEMENT FUSION (MICF) CONCEPT' (Nucl. Fusion 29 (1989) 1079)
}

\author{
T. KAMMASH, D.L. GALBRAITH \\ Department of Nuclear Engineering, \\ The University of Michigan, \\ Ann Arbor, Michigan, \\ United States of America
}

We agree that Eq. (115) as shown in our paper is incorrect. What was done in the paper, however, was to take a force balance on a spherical shell. If the shell falls between the radii $r=r_{0}$ and $r=r_{1}$, has a mass $m$ and an internal pressure $P_{1}$, and the face at $r_{0}$ experiences a pressure $P_{0}$ while that at $r_{1}$ sees a pressure $P_{2}$, then

$$
\begin{aligned}
& \frac{4 \pi}{3}\left(r_{1}^{2}-r_{0}^{2}\right) m\left\langle\frac{\partial u}{\partial t}\right\rangle=4 \pi r_{0}^{2} P_{0}-4 \pi r_{1}^{2} P_{2} \\
& +4 \pi\left(r_{1}^{2}-r_{0}^{2}\right) P_{1}
\end{aligned}
$$

where $\langle\partial u / \partial t\rangle$ is some appropriately chosen average value of the acceleration in the region. The differential form given in our Eq. (115),

$\mathrm{mN} \frac{\mathrm{du}}{\mathrm{dt}}=-\frac{\partial}{\partial \mathrm{r}}\left(\mathrm{r}^{2} \mathrm{P}\right)$

is obtained by neglecting the last term in Eq. (1), and is correct only if $r_{1} \approx r_{0}$. Since this term is always positive, its neglect certainly reduces the expansion (or increases the compression) calculated for the system. Neglecting this term was an oversimplification which we recognize should be (and has since been) removed from our model.
However, while this correction does reduce the calculated $\mathrm{Q}$ value somewhat, the reduction is nowhere near as drastic as suggested by Nakashima and Takabe. Making this change and no other changes in the model as reported in our paper, we find that the $\mathrm{Q}$ value is approximately halved. For the case quoted in the paper with an injection energy of $4.033 \mathrm{MJ}, \mathrm{Q}$ drops from 303.2 to 147.7 . This is still quite a large gain, making MICF a very attractive energy source. Thus, the differences in results between our model and that of Nakashima and Takabe obviously have some other cause.

In our model, the compression of the core results from a buildup of pressure in the innermost region of the metallic shell. This region is heated by bremsstrahlung from the core (and also by compressional heating) and becomes partially ionized. These freed electrons contribute to the pressure buildup; in the quoted calculation, the pressure in this region exceeds that in the core and halo regions at around 4-5 ns into the burn. It is this pressure difference which causes the observed compression of the core over the next $15 \mathrm{~ns}$. As we noted in our paper, the calculated gain $Q$ drops precipitously as the input energy falls below some critical minimum 
value. This critical value of $E_{\text {in }}$ varies with the pellet dimensions and appears to depend upon the details of the model used. Thus, it is not surprising if Nakashima and Takabe obtain much smaller $Q$ values than ours for at least some input energies.

The treatment in our model of the metallic shell is probably the area most in need of refinement. We have assumed that there is a region, of constant mass and uniform temperature, in which all of the bremsstrahlung energy is deposited uniformly, followed immediately by a region which receives no bremsstrahlung. Obviously, the true physical situation is much different. Conceivably, a better representation of this region of the shell would result in less, or possibly no, compression of the core.

Another issue is that some metallic ions will enter the halo (cold D-T fuel) region, and at least a few will migrate across the magnetic field into the core. These high- $\mathrm{Z}$ ions will increase the bremsstrahlung radiation, cooling these regions but possibly further heating the inner region of the metallic shell. If the latter occurs, the back pressure from this region would be still greater.

With as many complex interactions as occur in the MICF pellet, it is unlikely that any model can adequately represent everything. Probably, some features of the model used by Nakashima and Takabe are more appropriate than ours; it is also possible that our model is better in other respects. However, the differences in results are obviously not due solely to our oversimplification of the momentum equation. A final resolution of these differences almost certainly requires additional experimental data. We believe that MICF holds enough promise that such experiments should be undertaken. 